\section{HEART FAILURE \\ Non-transplant surgery for heart failure}

Stephen Westaby

Oxford Heart Centre, John Radcliffe Hospital, Headington, Oxford,

UK

hrombolysis and PTCA save lives during acute myocardial infarction, but incomplete or delayed reperfusion results in akinesia or dyskinesia. If more than $20 \%$ of the left ventricular circumference is dyskinetic, the remaining contractile cavity dilates to increase stroke volume. When more than $50 \%$ of the myocardium is impaired, increased wall tension (LaPlace's law) triggers progressive left ventricular failure with regression to myocyte fetal genetics and apoptosis. ${ }^{12}$ In Britain, most heart failure is caused by coronary artery disease, particularly in patients over 60 years old. There are now hundreds of thousands of patients with debilitating symptoms despite maximal medical treatment. Less than 300 cardiac transplants per year are undertaken in a labour intensive way by 10 separate units. In a short time, more palliated young patients with congenital heart disease will require these organs. Consequently, the treatment of older patients with coronary disease and idiopathic dilated cardiomyopathy requires a radical rethink.

Table 1 Relation between infarct size and mortality ${ }^{3}$

\begin{tabular}{lrc}
\hline & $\begin{array}{l}\text { Three year } \\
\text { mortality (\%) }\end{array}$ & p Value \\
\hline Myocardial infarction or scar $\geqslant 23 \%$ & 43 & 0.014 \\
Myocardial infarction or scar $<23 \%$ & 5 & 0.029 \\
$\mathrm{EF} \leqslant 43 \%$ & 38 & \\
$\mathrm{EF}>43 \%$ & 6 & 0.059 \\
$\mathrm{EF} \leqslant 43 \%$ without viable myocardium & 63 & \\
$\mathrm{EF} \leqslant 43 \%$ with viable myocardium & 13 &
\end{tabular}

Correspondence to: Mr Stephen Westaby, John Radcliffe Hospital, Headington, Oxford OX3 9DU

email: swestaby@ahf.org.uk

^For all patients with viable myocardium the three year mortality rate was $8 \%(80 \%$ had CABG). For patients with only fixed scar $>23 \%$ mortality rate was $50 \%(p=0.018)$. Only $40 \%$ had CABG with no difference in mortality with or without CABG. EF, ejection fraction.
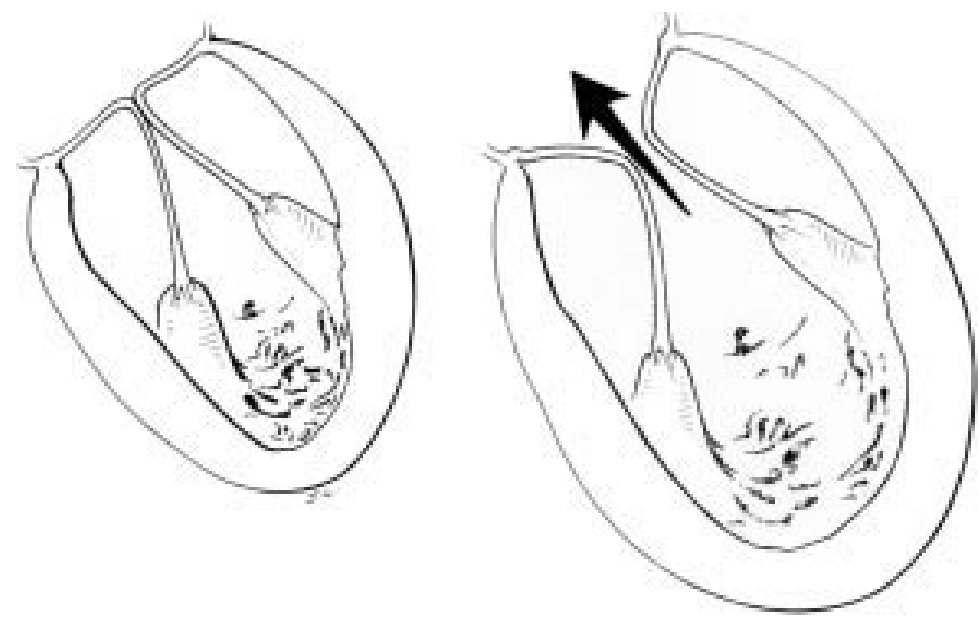

Figure 1: Progressive left ventricular dilatation causes mitral regurgitation and volume overload.
The following account of current and emerging surgical strategies for heart failure concentrates on those patients with left ventricular ejection fraction $(\mathrm{LVEF})<30 \%$, mean pulmonary artery pressure $>25 \mathrm{~mm} \mathrm{Hg}$, left ventricular circumferential akinesia or dyskinesia $>60 \%$, and left ventricular end diastolic volume (LVEDV) $>250 \mathrm{ml}$ (LVEDV index (LVEDVI) $>140 \mathrm{ml}$ ). Most of these patients are New York Heart Association (NYHA) functional class III or IV with medical treatment. In coronary disease the relation between infarct size and mortality has been well defined (table 1). ${ }^{3}$ From the coronary artery surgery study registry, five year survival for patients with $\mathrm{LVEF}<25 \%$ was $41 \%$ with medical treatment and $62 \%$ with surgery. ${ }^{4}$ For patients with dilated cardiomyopathy, mortality untreated is directly related to the severity of systolic dysfunction. Increased chamber sphericity and the presence of mitral regurgitation are markers of worse prognosis (one year mortality $54-70 \%$ ). In the failing heart, mitral regurgitation occurs secondary to annular dilatation, altered left ventricular geometry or papillary muscle dysfunction (fig 1). Volume overload causes progressive left ventricular and annular dilatation, worsened mitral regurgitation, and decreased survival.

\section{Coronary revascularisation in heart failure patients}

High risk coronary bypass is the most frequent conventional operation in heart failure patients. Incomplete myocardial infarction leaves viable but ischaemic myocardium within involved segments (flow/metabolism mismatch). Hibernating myocardium is an unstable substrate for postinfarction dysrhythmic events and mortality, independent of age or LVEF (event rate $43 \%$ v $8 \%$ for scar). ${ }^{5}$ Hibernating myocardium will recover contractile performance with reduced risk of dysrhythmia after coronary revascularisation, but for global improvement in left ventricular function, sufficient reversibly ischaemic territory must be present. Differentiation between reversible ischaemia and infarction is made on clinical grounds (angina which responds to sublingual nitrates) and objectively by positron emission tomography (PET) scan or dobutamine stress echocardiography. ${ }^{6}$ Heart failure patients without reversible ischaemia do not have an improved outlook with coronary bypass, and LVEF alone is a poor predictor of surgical

Cardiac transplantation is a very rare commodity.

- All transplant candidates with coronary disease should be assessed for hibernating myocardium then revascularisation.

- Those candidates with dilated cardiomyopathy should be considered for mitral repair. 
Left anterior descending coronary occlusion causes muscle loss in the apex, septum and anterolateral free wall.

In $20 \%$ of patients cardiac failure occurs within 15 months when the normal elliptical left ventricle loses its apex and becomes spherical.

The non-infarcted myocardium progressively dilates and further impairs function.

- When more than $50 \%$ of the circumference is asynergic surgical left ventricle restoration reduces left ventricular end systolic volume index (LVESVI) to $<80 \mathrm{ml} / \mathrm{m}^{2}$ and avoids progressive failure.

outcome when compared with the extent of reversible ischaemia. ${ }^{3}$ In fact, survival benefit from coronary bypass increases as LVEF decreases. Other contraindications to high risk coronary surgery are poor target vessels, a pulmonary artery pressure $>60 \mathrm{~mm} \mathrm{Hg}$, and significantly impaired right ventricular function.

The ethos for revascularisation of the failing ventricle can be summarised as simplicity, safety, and speed. The intra-aortic balloon pump is employed preoperatively. Only good target vessels to documented reversibly ischaemic myocardium are grafted to keep the cross clamp time short. Moderate and severe mitral regurgitation are corrected and full thickness scar excised where feasible. Those with access to left ventricular assist devices (LVADs) employ these to keep the perioperative mortality below $10-15 \% .^{8}$ Non-cardiac risk factors for death include great age, female sex, a history of hypertension or chronic obstructive airways disease, and the presence of peripheral vascular disease or renal impairment. In selected patients with hibernating myocardium but poor target vessels to some areas, concomitant transmyocardial laser revascularisation has been shown to improve survival. ${ }^{9}$

Useful data regarding patient selection and outcome for high risk revascularisation have emerged from transplant centres where end stage heart failure patients were selected out for myocardial revascularisation. Hausmann and colleagues in Berlin compared 225 revascularised transplant candidates with 231 others who received a donor organ. ${ }^{10}$ The important differences between the groups were the longer duration of symptoms, the presence of right heart failure, and a greater incidence of previous revascularisation in the heart trans-

High risk (5-15\% mortality) revascularisation is the treatment of choice for patients with ejection fraction $<20 \%$, reversible ischaemic cardiomyopathy and graftable target vessels.

- Contraindications to coronary bypass are pulmonary artery pressure $>60 \mathrm{~mm} \mathrm{Hg}$, right heart failure, and poor target vessels.
Table 2 Guidelines for coronary bypass versus

transplantation in end stage coronary artery disease $e^{10}$

\begin{tabular}{|c|c|}
\hline$C A B G$ & Transplant \\
\hline Prevailing hibernation & Prevailing scar \\
\hline $\begin{array}{l}\text { Short duration of heart } \\
\text { failure }\end{array}$ & Prolonged heart failure \\
\hline Low dose diuretics & High dose diuretics \\
\hline No right ventricular failure & $\begin{array}{l}\text { Chronic right ventricular } \\
\text { failure }\end{array}$ \\
\hline Stable cardiac output & Progressively lower output \\
\hline Cardia index $>2.01 / \mathrm{min} / \mathrm{m}^{2}$ & Cardiac index $<2.01 / \mathrm{min} / \mathrm{m}^{2}$ \\
\hline LVEDP $<24 \mathrm{~mm} \mathrm{Hg}$ & LVEDP > $24 \mathrm{~mm} \mathrm{Hg}$ \\
\hline Good target vessels & Poor vessels \\
\hline First operation & Previous revascularisation \\
\hline
\end{tabular}

LVEDP, left ventricular end diastolic pressure.

plant recipients. Operative risk in the coronary bypass group was significantly higher for those with a greatly increased left ventricular end diastolic pressure (LVEDP) $(>24 \mathrm{~mm} \mathrm{Hg}$ ), a low preoperative cardiac output $(<2.01 / \mathrm{min} /$ $\mathrm{m}^{2}$ ), and for patients in NYHA class IV. Hospital mortality was $7.1 \%$ for the coronary artery bypass graft (CABG) patients versus $18.2 \%$ in the transplant group. There was no significant difference in hospital mortality in patients with LVEF between 10-20\% versus those between $20-30 \%$. Survival for the CABG group was $78.9 \%$ after six years versus $68.9 \%$ in the transplant group. Reinvestigation of CABG patients showed a significant decrease in mean (SD) pulmonary artery pressure from 28.2 (4.7) $\mathrm{mm} \mathrm{Hg}$ to 21.2 (3.9) $\mathrm{mm} \mathrm{Hg}(\mathrm{p}<0.01)$. Pulmonary capillary wedge pressure fell from 19.2 (4.3) $\mathrm{mm} \mathrm{Hg}$ to 13.1 (2.8) $\mathrm{mm} \mathrm{Hg}$ $(\mathrm{p}<0.01)$. Left ventricular ejection fraction improved from a mean of $0.24(0.03)$ to 0.39 (0.06) $(\mathrm{p}<0.0001)$. Others have reported similar findings. ${ }^{112}$ Table 2 provides guidelines suggesting coronary bypass in preference to cardiac transplantation for patients with end stage coronary disease. Table 3 summarises the surgical treatment options in end stage ischaemic heart disease.

\section{Left ventricular restoration}

Patients with large left ventricular aneurysms gain symptomatic relief from simple linear aneurysmectomy. So called "ventricular restoration" has recently extended from scarred paradoxical segments to akinetic areas which were not previously thought suitable for surgery. ${ }^{13}$ The goal of surgical reversal of remodelling is to exclude the infarcted septum and free wall and reshape the left ventricle from globular to elliptical without critically reducing cavity volume. This improves global function

Table 3 Decision making in the surgery of end stage ischaemic heart disease

\begin{tabular}{ll}
\hline Condition & Intervention \\
\hline Reversible ischaemia LVESVI $<60 \mathrm{ml}^{\prime} \mathrm{m}^{2}$ & CABG alone \\
$\begin{array}{l}\text { Full thickness scar and left ventricular aneurysm } \\
\text { Akinetic/dyskinetic left ventricle LVESVI }>60\end{array}$ & Linear resection \pm CABG \\
$\mathrm{ml} / \mathrm{m}^{2}$ Reversible ischaemia & Surgical remodelling \pm CABG \\
Class III/IV mitral regurgitation & Mitral repair \pm CABG \\
No reversible ischaemia LVESVI $>100 \mathrm{ml} / \mathrm{m}^{2}$ & Left ventricular assist device or \\
Pulmonary hypertension (PAP $>70 \mathrm{~mm} \mathrm{Hg})$ & transplantation (no conservative option) \\
Right ventricular failure &
\end{tabular}

Right ventricular failure

LVESVI, left lentricular end systolic volume index (normal is $<30 \mathrm{ml} / \mathrm{m}^{2}$ ); CABG, coronary artery bypass grafting; PAP, pulmonary artery pressure. 


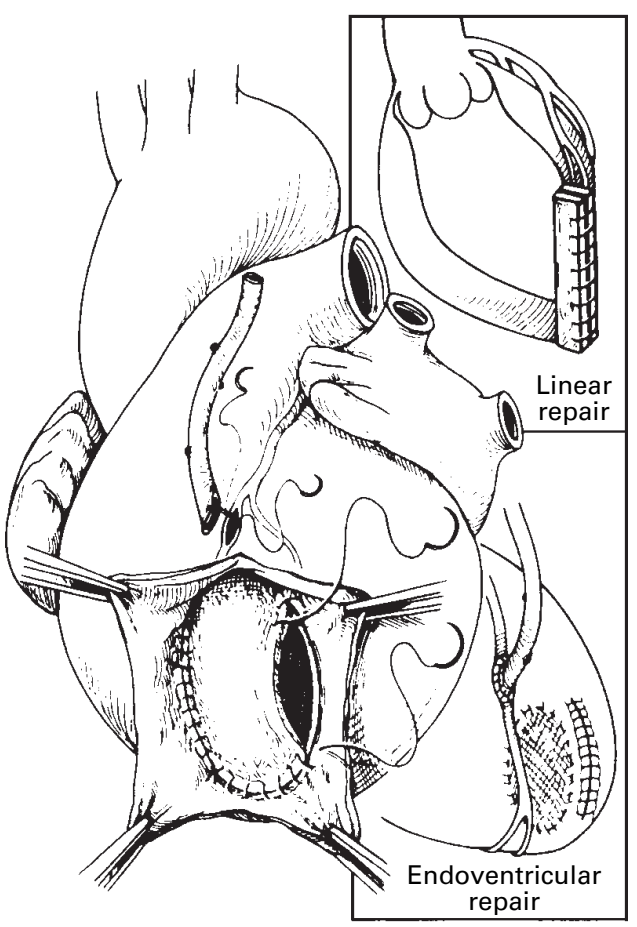

Figure 2: Left ventricular restoration by endoventricular patch repair (the Dor procedure) as opposed to simple linear aneurysmectomy.

and arrests progression of left ventricular failure. Ventricular dysrhythmias and mitral regurgitation are addressed during the same procedure.

In the Dor procedure, or the Buckberg modification, the left ventricle is opened through scar and subtotal endocardectomy (fig 2 ), performed over the septum and posterior wall. ${ }^{14}$ In the event of recurrent ventricular arrhythmias, cryotherapy is applied at the limits of the resection. The boundary between normal endocardium and scar is defined and a circumferential endoventricular (Fontan) circular suture passed between 1-2 cm outside the limit of healthy muscle. This circular constricting suture is tied to reduce the size of the left ventricle around a balloon inflated within the cavity to a diastolic volume of $50-70 \mathrm{ml} / \mathrm{m}^{2}$. The residual apical defect is then closed with a Dacron patch cut according to the circumference of the circular suture after removing the balloon. The technique restores an elliptical shape (fig 3) with improved function over the globular failing ventricle. Intraoperative echocardiography has shown a decrease in LVEDV from a mean of $194 \mathrm{ml}$ to $128 \mathrm{ml}(\mathrm{p}=0.001)$ and an improvement in LVEF from a mean of $29 \%$ to $41 \%$ $(\mathrm{p}=0.003)$. The operation can be performed in patients with very low LVEF $(<20 \%)$ and pulmonary hypertension with a hospital mortality of $12-18 \%$. This compares with a $3 \%$ mortality for those with ejection fraction $>30 \%$. Most patients improve to NYHA I or II, but $10 \%$ of survivors are not improved and about $25 \%$ have persistently raised pulmonary artery pressure through impaired diastolic compliance.

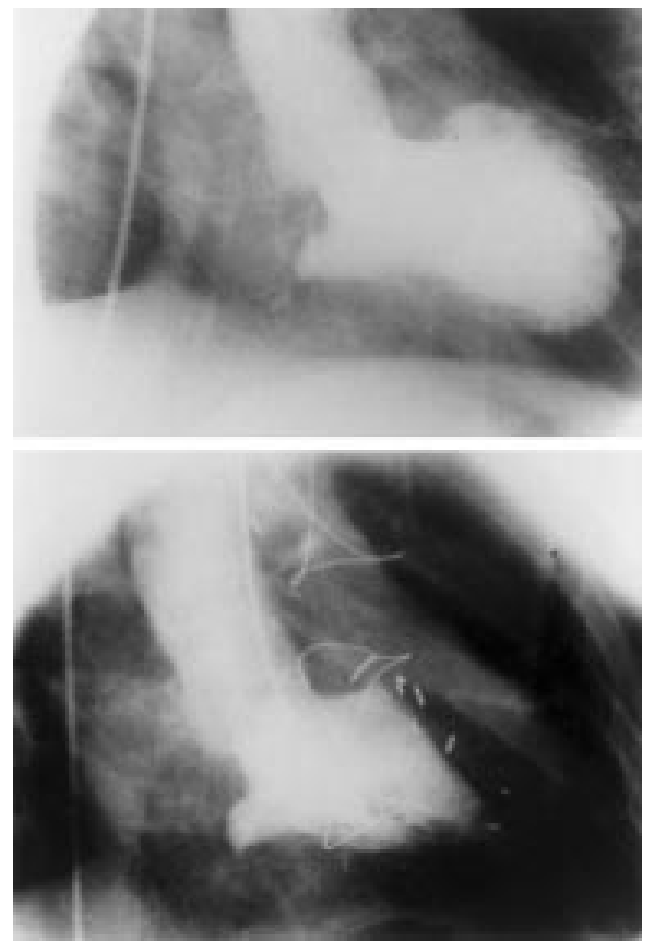

Figure 3: The Dor procedure restores an elliptical shape from the globular failing left ventricle (courtesy of Dr Vincent Dor).

Linear left ventricular aneurysm resection and the Dor procedure both improve remote myocardial function secondary to a reduction in wall tension. This "LaPlace" concept was expanded by Batista in his partial left ventriculectomy operation. ${ }^{15}$ The much hyped procedure was devised to reduce left ventricular volume and wall stress, thereby improving LVEF and symptomatic status. ${ }^{16}$ It was widely adopted as an alternative to transplantation without adequate guidelines or convincing information on sustainability or survival. The technique itself consists of a wedge resection of posterolateral left ventricular wall either between or including the papillary muscles. The incision begins at the apex of the ventricle and extends to within $2-3 \mathrm{~cm}$ of the mitral annulus (fig 4). Full thickness myocardial excision proceeds irrespective of the coronary anatomy and usually removes the obtuse marginal branches in the circumflex territory. The cavity is then reconstituted along its long axis with a continuous suture.

There is surprisingly little information on the amount of myocardium excised. The Cleveland Clinic group weighed the resected specimen, which ranged from 30-290 g (mean 96 g). ${ }^{17}$ The San Paolo group removed a posterolateral segment measuring 10.9 (2) $\mathrm{cm} \times 5$ $(0.8) \mathrm{cm}$, equivalent to about $20 \%$ of the left ventricular circumference. ${ }^{18}$ When the resection includes the base of one or both papillary muscles ( $88 \%$ of cases), the valve is either replaced with a prosthesis or the papillary muscles are reimplanted with transfixion sutures at the margins of the ventriculotomy (fig 5). If the mitral subvalvar apparatus is preserved, the free margins of the anterior and posterior leaflets are sewn together to produce 
- The goal of left ventricular restoration surgery (Dor procedure) is to reshape (from globular to elliptical) and reorganise the ventricle, not to reduce the volume. Reversible ischaemia, mitral regurgitation, and dysrhythmias should be addressed at the same time.

- Left ventricular end systolic volume index (LVESVI) is a strong predictor of death in heart failure (LVESVI $>60 \mathrm{ml} / \mathrm{m}^{2}$ carries a one year mortality of $33 \%$ ).

- Patients with a good outcome from the Dor operation have an LVESVI $>40 \mathrm{ml} /{ }^{2}$.

- Coronary bypass alone improves ejection fraction only if the preoperative LVESVI is $<100 \mathrm{ml} / \mathrm{m}^{2}$.

- Linear left ventricular remodelling (the Batista operation) is unpredictable with an unacceptable early failure rate and late mortality.

a double channelled mitral valve (Alfieri method) ${ }^{19}$ (fig 6). Even published hospital mortality has been prohibitive, ranging from $1.9-27 \%$ with an average of $17.4 \% .{ }^{20}$ Low hospital mortality has been achieved only with the aid of long term LVAD support (20\% of patients), and cardiac transplantation.

In survivors, there is a significant decrease in both end diastolic and end systolic volume indices. While LVEF initially improves, restudy at 12 months fails to show significant differences between preoperative LVEF (17.7 (4.6)) and late LVEF (23.7 (6)) in matched patients. The suggested mechanism for improvement in LVEF is reduction of systolic wall stress rather than a change in contractility. There is an inverse relation between the decrease of circumferential end systolic stress and increase in LVEF. In McCarthy's series, mean LVEF improved from $13 \%$ to $21 \%$ and peak oxygen consumption from $11 \mathrm{ml} / \mathrm{kg} / \mathrm{min}$ to $16 \mathrm{ml} / \mathrm{kg} /$ min at 12 months. ${ }^{17}$ Twelve month survival at the Cleveland Clinic was $80 \%$ though LVADs were required for bridge to transplantation in $16 \%$ of patients. However, freedom from heart failure of any cause (relisting for transplant, death or class IV symptoms) was only $50 \%$ by 12 months and $38 \%$ at two years.

Though the reduced ventricular geometric dimensions may be sustained up to 12 months (fig 7), pump function begins to deteriorate after six months. The first sign is a rise in left atrial pressure. The discrepancy between geometry and sustainability of mechanical function is attributed to the fact that mass reduction causes changes in diastolic compliance.

Though late data are scarce, we defined a $16 \%$ mortality from all reported series through progressive heart failure $(38 \%)$, sudden or arrhythmic death (38\%), stroke, transplant heart failure, sepsis or hepatic failure. ${ }^{20}$ The procedure has been abandoned in ischaemic cardiomyopathy through a prohibitive incidence of fatal dysrhythmias caused by stretch-
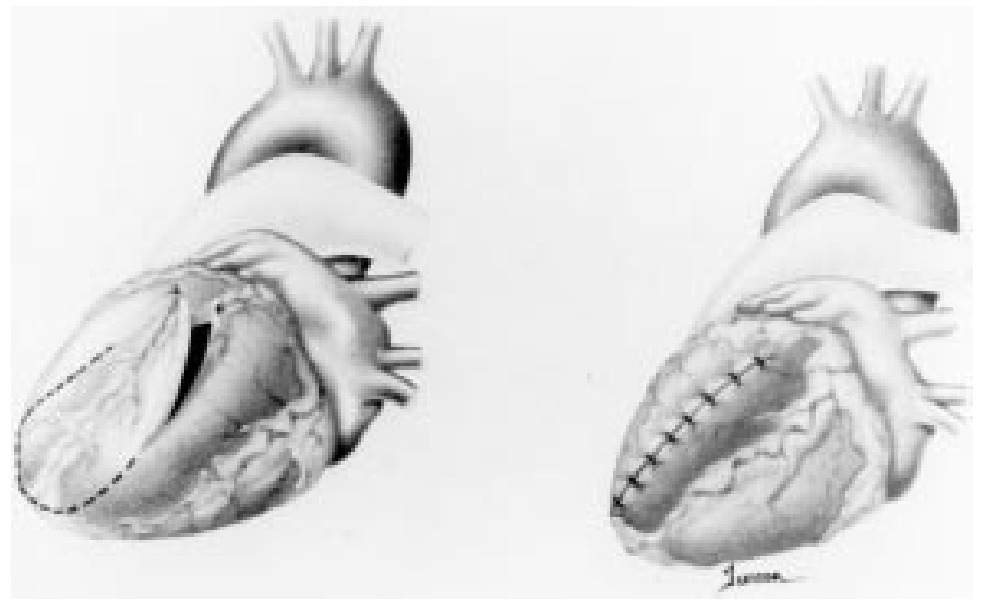

Figure 4: Partial left ventriculectomy (the Batista operation).
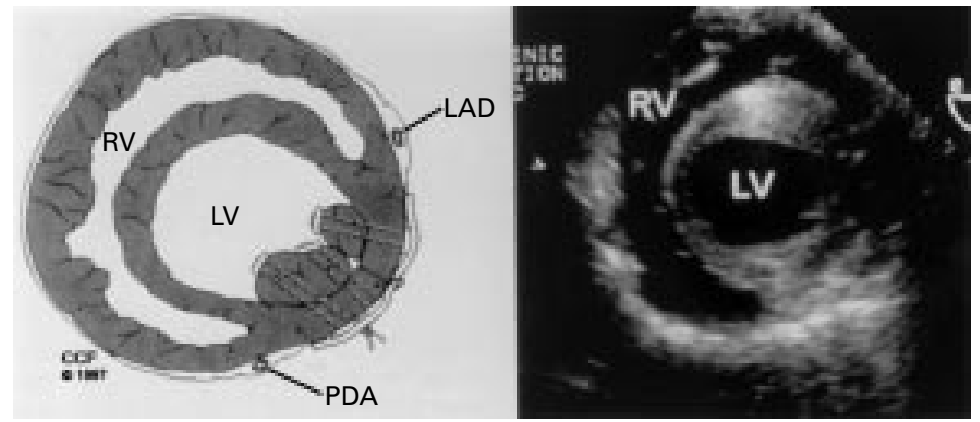

Figure 5: Diagrammatic and echocardiographic illustration of the partial left ventriculectomy operation. The mitral subvalvar apparatus is preserved by reimplanting the papillary muscles at the margins of the ventriculotomy.

ing of the scar tissue. ${ }^{21}$ In reality, most centres who enthusiastically embraced partial left ventriculectomy have now radically cut back operating only on highly selected dilated cardiomyopathy patients. With emerging alternatives the Batista operation is destined to join skeletal muscle cardiomyoplasty in the dustbin of heart failure operations.

\section{Management of mitral regurgitation in heart failure patients}

As the failing left ventricle dilates the papillary muscles are displaced, the coaptation of the mitral valve leaflets is decreased, and a central jet of mitral regurgitation appears (fig 1). Mitral regurgitation leads to more volume

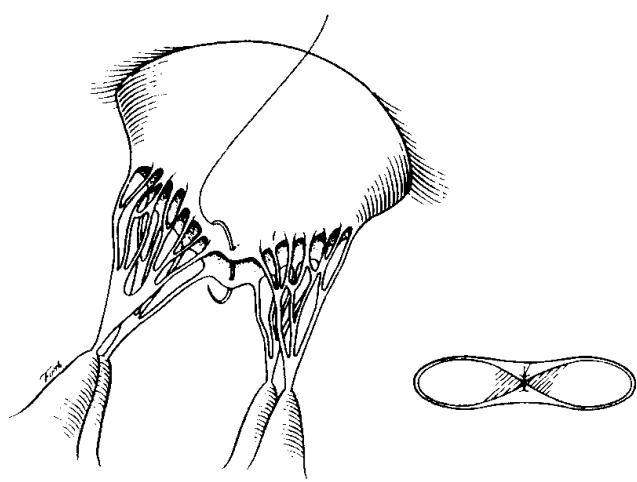

Figure 6: The Alfieri suture for mitral regurgitation. This creates a double channel mitral orifice. 


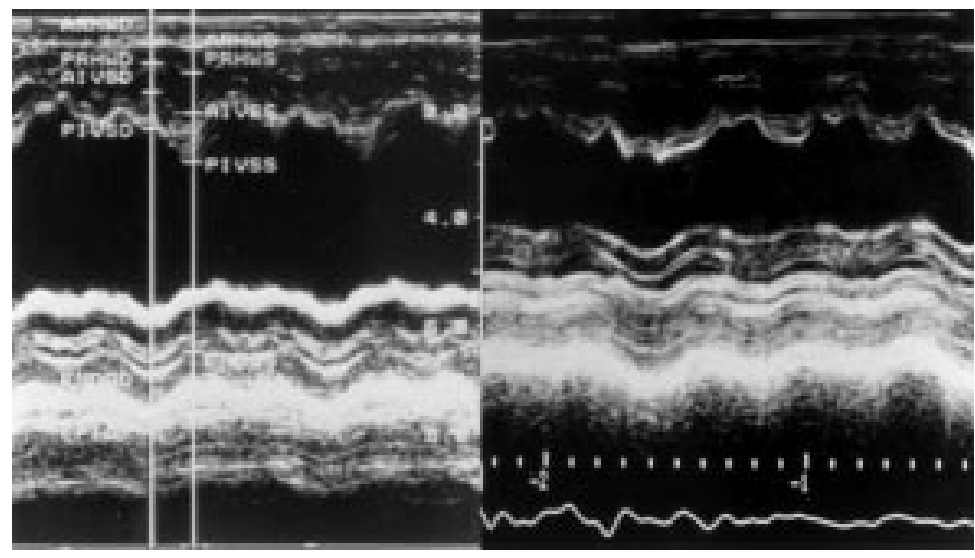

Figure 7: One dimensional (M mode) echocardiography showing dramatically improved left ventricular function in an infant six months after partial left ventriculectomy for ischaemic cardiomyopathy in anomalous left coronary artery from the pulmonary artery.

Table 4 Mitral valve repair in dilated cardiomyopathy. Left ventricular function and flow before and after annuloplasty

\begin{tabular}{lcccc}
\hline Variable & Preoperatively & Postoperatively & \% change* & p Value \\
\hline End diastolic volume (ml) & $335(107)$ & $307(103)$ & $18(9)$ & 0.06 \\
End systolic volume (ml) & $227(101)$ & $237(98)$ & $-15(14)$ & 0.03 \\
Stroke volume (ml) & $58(13)$ & $70(21)$ & $+12(10)$ & 0.02 \\
Ejection fraction (\%) & $18(5)$ & $24(10)$ & $+31(24)$ & 0.03 \\
Mitral inflow (1/mm) & $12.4(5.3)$ & $5.4(0.5)$ & $-49(22)$ & 0.02 \\
Forward cardiac output $(1 / \mathrm{min})$ & $3.2(1.0)$ & $4.7(0.9)$ & $+52(38)$ & 0.01 \\
Regurgitant volume $(1 / \mathrm{min})$ & $9.2(5.4)$ & $0.8(0.6)$ & $88.6(10.1)$ & 0.01 \\
Regurgitant fraction $(\%)$ & $70(14)$ & $15(11)$ & $-79(15)$ & $<0.001$
\end{tabular}

Values are mean (SD). ${ }^{\star}$ Percentage change from preoperative study at 4-6 months postoperatively. NYHA class fell significantly from $3.9(0.4)$ to $1.8(0.5)(\mathrm{p}<0.001)$.

Reproduced from Bolling et al. F Thorac Cardiovasc Surg 1998;115: 381-8. with permission of the publisher.

overload of the already dilated left ventricle. Reports of prohibitive operative mortality for mitral valve replacement in dilated cardiomyopathy patients in the early 1980s suggested that the failing ventricle deteriorates further, if the "blow off" into the left atrium is removed. However, new information shows that mitral valve repair (or replacement) with preservation of the subvalvar apparatus carries low perioperative mortality, good medium term survival, and symptomatic relief through improvement in cardiac index..$^{22}$

There are now a number of clinical situations where mitral valve repair with or without revascularisation improves outlook for the heart failure patient.

These include:

- Ischaemia manifest by angina and variable mitral regurgitation which becomes significantly worse during an acute ischemic episode, causing dyspnoea at rest or left ventricular failure with pulmonary oedema.

- Acute myocardial ischaemia or infarction located inferobasally (right coronary or dominant circumflex distribution) which causes sudden posteromedial papillary muscle dysfunction and mitral regurgitation.

- Acute catastrophic pulmonary oedema caused by papillary muscle rupture (inferobasal in $75 \%$ of cases) several days after acute myocardial infarction.

- Chronic progressive dyspnoea (NYHA III or IV) associated with previous myocardial infarction, an enlarged dysfunctional left ventricle, and varying degrees of pulmonary hypertension. This comprises the largest group.

- Patients with idiopathic dilated cardiomyopathy and annular dilatation producing moderate to severe mitral regurgitation through inadequate leaflet coaptation.

The recommended threshold for mitral repair in ischaemic regurgitation is a left ventricular end systolic volume index $>80 \mathrm{ml} /$ $\mathrm{m}^{2}$ or a calculated regurgitant fraction $>50 \%$ of the forward LVEF. Patients with angina, good target vessels, mild to moderate mitral regurgitation, and reversible ischaemia posterolaterally on the PET scan can be treated by revascularisation alone. Should valve replacement prove necessary, as much of the subvalvar apparatus as possible should be conserved to maintain left ventricular geometry and function. Division of all chordae tendonae is accompanied by a $47 \%$ reduction in $\mathrm{LVE}_{\text {max }}$.

Ischaemic mitral regurgitation is a functional problem of unsuccessful coordination of the entire mitral apparatus rather than simple failure of a single papillary muscle. Two techniques have provided symptomatic improvement in this condition. Firstly, mitral annuloplasty with significant undersizing of the valve ring greatly increases leaflet coaptation. ${ }^{23}$ Systolic anterior motion (SAM) is avoided because of widening of the aortomitral angle and increased left ventricular size. The undersized valve ring acutely remodels the base of the myopathic heart, helping to re-establish an ellipsoid shape to the left ventricle. Second, and simpler, is the Alfieri stitch. This can be performed either centrally (fig 6) or towards the side of the ischaemic papillary muscle.

Bolling has shown the important effect of mitral repair in patients with end stage dilated cardiomyopathy. ${ }^{22}$ All had severe left ventricular systolic dysfunction with preoperative LVEF ranging from 8-25\% (mean 16 (3)\%). The average duration of cardiomyopathy was 4 (6) years (range 0-16). All patients underwent remodelling ring annuloplasty with an undersized flexible ring. Half had tricuspid annuloplasty. Hospital mortality was $<2 \%$ while 12 and 24 month survival were $82 \%$ and $72 \%$. All patients were restored to NYHA class I or II with mean postoperative LVEF of $26 \%$. Peak exercise $\mathrm{VO}_{2}$ max rose from a mean of 14.5 to $18.6 \mathrm{ml} / \mathrm{kg} / \mathrm{min}$. Echocardiography at two years showed a pronounced reduction in sphericity, regurgitant volume, and regurgitant fraction. LVEF, end diastolic and end systolic volumes were all improved (table 4).

\section{Mechanical blood pumps}

Ideal treatment for chronic refractory heart failure should be reliable, cost effective, easy to implement, and capable of providing a physiological level of circulatory support. Existing cardiac support devices such as the pacemaker and implantable defibrillator are already widely accepted for patients of all ages. Within the next 10 years a user friendly miniaturised LVAD is destined to become the treatment of 
choice to relieve symptoms and prolong life in older heart failure patients.

Total heart replacement became virtually redundant when it was clear that more than $90 \%$ of patients could be sustained with left ventricular support alone. Only those with advanced right ventricular pathology or fixed pulmonary hypertension require biventricular support. Those LVADs currently used for bridge to transplantation have their origins in the 1970s and can be regarded as first generation blood pumps. The Novacor (Baxter Health Care, California, USA) and ThermoCardio Systems (Woburn, Massachusetts, USA) LVADs consist of a blood sac in series with the native left ventricle and compressed by a pusherplate mechanism, either electrically or pneumatically driven. ${ }^{24}{ }^{25}$ Bioprosthetic heart valves dictate the direction of flow. This mechanism mimics the native left ventricle by providing pulsatile stroke volume with either variable or fixed pump rate. The patients own left ventricle is completely offloaded so that the aortic valve does not open. While large external pneumatic consoles have been replaced by implantable electric systems with portable control and power source, the serious problem of LVAD size, noise, driveline infection, and thromboembolism persist. These devices are unsuitably large for most female patients or children. Nevertheless, some bridge to transplant patients have survived with acceptable quality of life for up to four years. ${ }^{26}$

The new axial flow impeller pumps are the next generation of artificial hearts. ${ }^{27}$ In animal studies these compact, silent, non-pulsatile blood pumps provide up to 6 litres flow per minute, without significant haemolysis or thromboembolism. The thumb sized Jarvik 2000 heart fits within the apex of the failing left ventricle and pumps blood to the descending thoracic aorta (fig 8). ${ }^{28}$ The impeller supported by blood immersed microceramic bearings revolves at up to $18000 \mathrm{rpm}$ accelerating blood so rapidly through a narrow channel that the cellular components remain undamaged. The controller and batteries are the size of a portable telephone and fit easily onto a normal belt. While transcutaneous power induction is under development, we have devised an infection resistant skull mounted percutaneous titanium pedestal for the first human implants. ${ }^{29}$ The extracardiac NASA/de Bakey axial flow pump has already been tested for bridge to transplantation in humans with mixed results owing to mechanical problems.

Other ingenious blood pumps with magnetically suspended rotors (without bearings) are under development. These next generation, fully implantable miniature artificial hearts greatly increase the future scope of circulatory support, but mechanical reliability and freedom from complications remains to be established. We must also define the effects of chronic non-pulsatile blood flow, though the recovering native heart will transmit a pulse through the device. We anticipate that the cost of a blood pump will be less than that of multiple hospital admissions for stabilisation during the last year of the patient's life. The device will

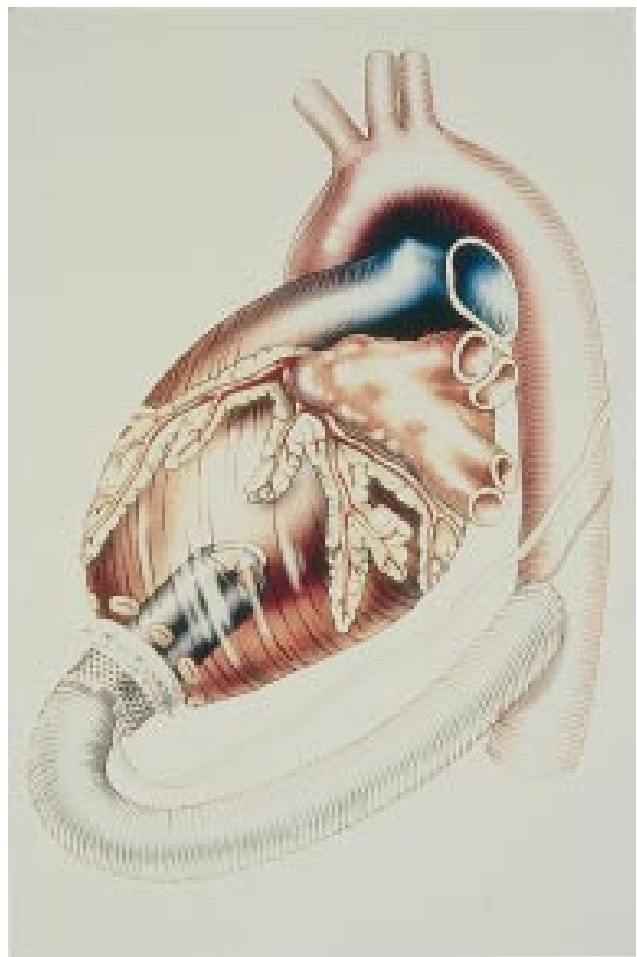

Figure 8: The intraventricular "Jarvik 2000 Heart", an axial flow impeller pump which rotates at between 8000 and $18000 \mathrm{rpm}$ without damaging the blood.

- A realistic blood pump for long term circulatory support must be unobtrusive, silent, mechanically reliable with economic energy consumption, non-thrombogenic, free from constant infection risk and able to provide between 3-10 litres flow per minute without haemolysis. Such devices are now available for human implant.

- In the next 10 years blood pumps will be used for heart failure in the same way that pacemakers and implantable defibrillators are used for dysrhythmia.

replace most drug treatment and require only a maintenance dose of warfarin.

\section{Reversal of remodelling with an LVAD}

One of the revelations of the past five years is the effect of chronic left ventricular offloading on the remodelled left ventricle. ${ }^{30}$ Reduction in cardiac workload by strict prolonged bed rest is known to result in functional and symptomatic improvement, though the benefits are limited by the adverse effects of inactivity on the skeletal muscles, vascular tone, and autonomic nervous system. However, when myocardial rest is accompanied by whole body exercise training the combined benefits become apparent. These are the conditions achieved during long term bridge to transplantation with the Thermocardio Systems and Novacor LVADs. The improved systemic blood flow reverses multisystem organ failure and enables resumed physical activity, while the left ventricle is com- 
pletely offloaded. Renal and hepatic failure improve whereas serum aldosterone concentrations, plasmin renin activity, atrial natriuretic peptide, and norepinephrine concentrations revert to normal. ${ }^{31}$ In dilated cardiomyopathy patients bridged to transplantation, left ventricular size regresses with normalisation of pressure volume relations, resolution of left ventricular hypertrophy, and decreased myocytolysis, fibrosis, and apoptosis. ${ }^{32}$ Comparison of myocardial biopsies taken at the time of LVAD implantation, then later during transplantation, show regression of myocyte hypertrophy with normalisation of calcium, phospholipid, and fatty acid metabolism. Realisation that recovering hearts were being discarded by transplantation, together with the incontrovertible shortage of organ donors, led to the concept of bridge to myocardial recovery. ${ }^{33}$

Although the scope for bridge to recovery is obvious, there are certain requirements before this strategy has a chance for success. The first is a user friendly LVAD for patients of all sizes. This must be simple to implant and remove without the risk of driveline infection and easy for the patient to control. The axial flow pumps with a skull mounted percutaneous pedestal or transcutaneous power induction are promising in this respect. Second, the LVAD should be employed before diffuse fibrosis renders reversal of ventricular remodelling unachievable. ${ }^{34}$ Strategies to promote myocardial recovery with drugs, growth factors, gene therapy, and inhibitors of apoptosis are under investigation and markers for sustainability of recovery explored. ${ }^{35}$ In this respect, the Berlin group has used the disappearance from the serum of the autoantibody against the $\beta_{2}$ adrenergic receptor, suggesting that this reflects abatement of the immune process causing functional impairment. ${ }^{36}$

Clinical reports of bridge to recovery demonstrates the importance of myocardial pathology. Patients with acute myocarditis who are in terminal decline can be supported with an LVAD or BIVAD until resolution of the inflammatory process. Even those who require external cardiac massage and conventional cardiopulmonary bypass to sustain life during LVAD implantation can be restored to near normal cardiac function.

In a study of weaning of dilated cardiomyopathy patients from chronic left ventricular support, Muller compared factors which distinguished those with well sustained recovery from others who slipped back into heart failure. ${ }^{35}$ Patients with long lasting recovery were younger, had a shorter history of heart failure, had a more rapid improvement in cardiac performance, and needed a shorter duration of LVAD support before cardiac indices justified device removal. The duration over which the autoantibodies disappeared from the serum was shorter (8.8 (2.3) weeks $v 9.7$ (3.3) weeks, $p=0.0027)$ in patients with sustainable recovery. Not different were mean age (41.5 (9.9) years $v 50.3$ (11.2)), mean LVED diameter at the time of device placement (75.2 (7.9) $\mathrm{mm}$ v 78.7 (4.8) $\mathrm{mm}$ ), LVEF (14.8 $(2.9) \%$ \% $17.0(2.3) \%)$, and mean LVED diameter two months after LVAD placement (53.7 (6.2) $\mathrm{mm} v 55.6$ (7.2) mm). The Berlin group concluded that an LVAD offloading period of between 8-10 weeks was optimum in patients destined for sustainable recovery, and that longer support could lead to myocyte atrophy. Others have reservations about this limited duration and report success for much longer periods. ${ }^{1}$

\section{Summary}

The scope of heart failure surgery is developing rapidly but relies increasingly upon expensive diagnostic techniques and mechanical circulatory support. The major issues are not ethical but economic. New miniature axial flow and centrifugal blood pumps are emerging from bioengineering laboratories and will eventually be used as frequently for heart failure as is the pacemaker for rhythm problems. In the future, new drugs, gene therapy, and autogenous myocyte culture will promote left ventricular repair during circulatory support, thereby freeing the limited number of donor organs for younger complex congenital hearts.

1. Klein M, Herman M, Gorlin R. A haemodynamic study of left ventricular aneurysm. Circulation 1967;35:614-30.

2. Olivetti G, Abbi R, Quaina F, et al. Apoptosis in the failing human heart. N Engl J Med 1997;336:1131-41.

3. Yoshida F, Gould KL. Quantitative relation of myocardia infarct size and myocardial viability by positron emission tomography to left ventricular ejection fraction and 3 year mortality with and without revascularisation. J Am Coll Cardiol 1993;22:984-97.

4. Emond M, Mock MB, Davis KB, et al. Long-term survival of medically treated patients in the coronary artery surgery study (CASS). Circulation 1994:90:2645-57.

5. Lee KS, Marwick TH, Cook SA, et al. Prognosis of patients with left ventricular dysfunction, with and without viable myocardium after myocardial infarction. Relative efficacy of medical therapy and revascularisation. Circulation 1994;90:2687-94

- After myocardial infarction, residual viable but ischaemic myocardium is an unstable substrate for further events. This study shows surgical revascularisation reduces the risk of these events, though age and the severity of left ventricular function remain the best predictor of death.

6. Williams MJ, Odabashiar J, Lauer MS, et al. Prognostic value of dobutamine echocardiography in patients with left ventricular dysfunction. J Am Coll Cardiol 1996;27:132-9.

7. Di Carli MF, Davidson M, Little R, et al. Value of metabolic imaging with positron emission tomography for evaluating prognosis in patients with coronary artery disease and left ventricular dysfunction. Am J Cardiol 1994;73:527-33.

8. Louie HW, Laks H, Milgalter E, et al. Ischemic cardiomyopathy. Criteria for myocardial revascularisation and cardiac transplantation. Circulation 1991;84:(suppl III):III290-5.

9. Allen KB, Delrossi EJ, Realyvasquez F, et al. Transmyocardial revascularisation combined with coronary artery bypass grafting versus bypass grafting alone: a prospective randomized mult
Cardiovasc Surg In press.

10. Hausmann $\mathbf{H}$, Topp $\mathbf{H}$, Siniawski $\mathbf{H}$, et al. Decision making in end stage coronary artery disease: revascularisation or heart transplantation. Ann Thorac Surg 1997:64:1296-1302.

- This paper reviews the outcomes of patients subject to high risk coronary revascularisation or transplantation in a pool of end stage heart failure patients referred for pool of end stage heart failure patients referred for treated by coronary bypass had a better long term outlook treated by coronary bypass had a better long term outlook
than those transplanted. The article outlines which patients should undergo coronary bypass in preference to transplantation.

11 Kaul TK, Agnihotri AK, Fields BL, et al. Coronary artery bypass grafting in patients with an ejection fraction of twenty per cent or less. J Thorac Cardiovasc Surg 
12. Mickleborough LL, Maruyama H, Yasushi T, et al. Results of revascularisation in patients with severe left ventricular dysfunction. Circulation 1995;92:(suppl 2):73-9.

13. Buckberg GD. Defining the relationship between akinesia and dyskinesia and the cause of left ventricular failure after anterior infarction and reversal of remodelling to restoration. J Thorac Cardiovas Surg 1998;116:47-9.

14. Dor V, Sabatier M, DiDonato M, et al. Efficacy of endoventricular patch plasty in large post infarction akinetic scar and severe left ventricular dysfunction: comparison with a series of large dyskinetic scars. J Thorac Cardiovasc Surg 1998;116:50-9.

- The Dor procedure remodels the globular failing left ventricle back to an elliptical shape with improved function. This paper describes the functional improvement and outlook for those patients with ischaemic cardiomyopathy.

15. Batista RJV, Nery P, Bocchino L, et al. Partial left ventriculectomy to treat end stage heart disease. Ann Thorac Surg 1997;64:634-8.

16. Dickstein ML, Spotnitz HM, Rose EA, et al. Heart reduction surgery: An analysis of the impact on cardiac function. J Thorac Cardiovasc Surg 1977;113:1032-40.

17. McCarthy PM, Starling RC, Wong J, et al. Early results with partial left ventriculectomy. J Thorac Cardiovasc Surg 1997:114:755-65.

18. Moreira LFP, Stolf NAG, Bocchi EA, et al. Partial left ventriculectomy with mitral valve preservation in the treatment of patients with dilated cardiomyopathy. J Thorac Cardiovasc Surg1998:115:800-7.

19. Fucci L, Sandrelli L, Pardini A, et al. Improved results with mitral valve repair using new surgical techniques. Eur $J$ Cardiothorac Surg 1995;9:621-7.

20. Katsumata T, Westaby S. An objective appraisal of partial left ventriculectomy for heart failure. Journal of Congestive Heart Failure and Circulatory Support 1999;1:97-106.

21. Bach DS, Bolling SF. Early improvement in congestive heart failure after correction of secondary mitral regurgitation in end-stage cardiomyopathy. Am Heart $J$ 1995;129:1165-70.

22. David TE. Techniques and results of mitral valve repair for ischemic mitral regurgitation. J Cardiac Surg 1994;9(suppl):274-7.

23. McCarthy PM, Portner PM, Tobler HG, et al. Clinica experience with the Novacor ventricular assist system. $J$ Thorac Cardiovasc Surg 1991;102:573-81.

24. Frazier OH, Rose EA, MacMannus Q, et al. Multicentre clinical evaluation of the Heartmate 1000 IP left ventricular assist device. Ann Thorac Surg 1992;53:1080-90.

25. Frazier $\mathrm{OH}$, Rose EA, McCarthy $\mathbf{P}$, et al. Improved mortality and rehabilitation of transplant candidates treated with a long term implantable left ventricular assist system. Ann Thorac Surg 1995;222:327-38.
26. Belland S, Jeevanandam V, Eiser $\mathbf{H}$. Reduced myocardial matrix metaloproteinase expression as a result of sustained mechanical support with left ventricular assist devices in patients with severe dilated cardiomyopathy [abstract]. J Heart Lung Transplant 1998;17:84.

27. Westaby S, Katsumata T, Houel R, et al. Jarvik 2000 Heart-potential for bridge to myocyte recovery. Circulation 1998;98:1568-74.

28. Jarvik RK, Westaby S, Katsumata T, et al. LVAD power delivery. A new percutaneous approach to avoid infection. Ann Thorac Surg 1998;65:470-3.

- Power line infection is one of the restricting elements which must be overcome before LVADs can be used with widespread efficacy. This short paper describes an innovative method to avoid drive line infection pending the development of transcutaneous power induction.

29. Levin HR, Oz MC, Cherr JM, et al. Reversal of chronic ventricular dilation in patients with end stage cardiomyopathy by prolonged mechanical offloading. Circulation 1995;91:2717-20.

30. James KB, McCarthy PM, Thomas JD, et al. Effect of the implantable left ventricular assist device on neuroendocrine activation in heart failure. Circulation 1995;92(suppl II):191-5.

31. Frazier $\mathrm{OH}$, Benedict CR, Radovancevic B, et al. Improved left ventricular function after chronic left ventricular unloading. Ann Thorac Surg 1996;62:675-82.

32. Mann DL, Willerson MD. Left ventricular assist devices and the failing heart. A bridge to recovery, a permanent assist device or a bridge to far? Circulation 1998;98:2367-9.

33. Mancini DM, Beniaminovitz A, Levin $\mathbf{H}$, et al. Low incidence of myocardial recovery after left ventricular assis device implantation in patients with chronic heart failure. Circulation 1998;98:2383-9.

34. Kirshenbaum LA, de Moissac D. The bcl-2 gene product prevents programmed cell death of ventricular myocytes. Circulation 1997;96:158-5.

35. Muller J, Wallukat G, Weng Y, et al. Weaning from mechanical support in patients with dilated cardiomyopathy. Circulation 1997:96:542-9.

36. Hetzer R, Loebe M, Potapov EV, et al. Circulatory support with pneumatic paracorporeal ventricular assist device in infants and children. Ann Thorac Surg

1998:66:1498-506.

- This paper from the Berlin Heart Institute describes their experience of mechanical circulatory support in infants and young children. The very important finding was that infants with myocarditis and terminal circulatory shock could be resuscitated, supported for 2-3 weeks, and eventually have normal hearts after removal of the device. 